\title{
Texturing 3D-Reconstructions of the Human Brain With EEG-Activity Maps
}

\author{
Leonid I. Dimitrov*
}

Institute of Information Processing, Austrian Academy of Sciences, Vienna, Austria

\begin{abstract}
A method for representing the results of statistical EEG analysis in a visually comprehensive and concentrated way is presented. It consists of a combination of scattered data interpolation, texture mapping, and volume rendering techniques. First, starting with an EEG study of the proband in question, a smoothly interpolated rectangular map of the EEG findings in the $(\varphi, \vartheta)$-domain $(\varphi$ and $\vartheta$ denote spherical coordinates) is calculated. Then, using the data from an accompanying MRI scan, volume-rendered representations of the brain with a pseudo-colored cortical surface, vividly depicting the precalculated EEG-activities, are produced. Custom-developed software was used for interpolating the EEG maps from the scattered data samples, as well as for mapping and rendering them together with the brain volume data. The algorithm which achieves the scattered data interpolation is a genuine development employing Delaunay triangulation and a modification of the well-known rotation-invariant Phong interpolation. The pictures produced by the presented method are currently being utilized in neurophysiology for detecting correlations between EEG-parameters, cortical morphology, and underlying mental activities. Hum. Brain Mapping 6:189-202, 1998. @ 1998 Wiley-Liss, Inc.
\end{abstract}

Key words: EEG; MRI; scattered data interpolation; texture mapping; volume visualization

\section{INTRODUCTION}

\section{Motivation}

Functional brain mapping presents itself today as a steadily growing field with many open questions and potential applications and naturally attracts the attention of research and industry likewise.

The modern neuroimaging modalities applied in this field, such as positron emission tomography (PET) or functional magnetic resonance imaging (fMRI) unquestionably exhibit powerful possibilities and great

Contract grant sponsor: Austrian Forschungsförderungsfonds; Contract grant number: P8189-MED.

*Correspondence to: Leonid I. Dimitrov, Institute of Information Processing, Austrian Academy of Sciences, Sonnenfelsgasse 19/2, A-1010 Vienna, Austria. E-mail: Leonid.Dimitrov@oeaw.ac.at Received for publication 25 April 1997; accepted 7 April 1998 potential for further insights into the workings of the healthy, normally functioning brain. However, it can't be overlooked that certain difficulties in connection with their application exist, e.g., low temporal resolution, heavy prohibitive equipment, and high cost, which render them inadequate for studies of the everyday functioning of the brain under normal as opposed to artificial, test-like conditions.

At the same time, they tend to overshadow an older, but nonetheless extremely useful technique for recording and studying the rapidly changing patterns of brain activity underlying mental function, viz. electroencephalography (EEG). The indisputable advantages of this traditional neuroimaging technique, such as high temporal resolution in the millisecond-range, ease of application and, last but not least, low cost, seem to make it an ideal candidate for combining with the above-mentioned imaging modalities and thus overcoming their deficiencies. 
One of the obvious applications lies in the possibility to advantageously use a combination of these technologies and try to answer the old question about the relationship between structure and function of the human cortex. Endeavors to find such relationships have been a major research topic for many years, but ever since these endeavors were initiated, a major obstacle has been the poor spatial resolution of scalp-recorded EEG and the great difficulties to localize precisely the underlying (cortical) sources from the EEG recordings alone.

The major difficulty seems to lie in the fact, that what we record at the scalp is the sum of many contributions from possibly many of the EEG generators, after the conduction and inevitable distortion (blurring) of the electric fields through the tissues of the head. One way of dealing with this fact is the attempt to calculate back from the recorded samples/scalp potential map to their probable origins/source distribution, which includes both the development of a suitable generation and propagation model, as well as its simulation and inversion. This constitutes the so-called inverse problem. Two directions of research are dipole localization and spatial deconvolution [see Nunez, 1995]. In order to solve it, elaborate techniques of deblurring involving Finite or Boundary Element Models have been developed [Gevins et al., 1991, 1994]. These provide us then with a spatially better resolved, but still somewhat disconnected distribution of the electrical activities around the cortex without a direct relation to the underlying anatomical structures. In order to be able to judge better the measured and evaluated (possibly deblurred) cortical activities, it would be highly desirable to visualize them in their natural environment where they emerged in the first place, namely on the cortex. The emphasis here lies on visualize which shouldn't implicate that this visualization process makes the spatial deblurring obsolete, on the contrary-the better the input data is, the more conclusive and expressive the visualization will be. What we achieve through the visualization is a unified data representation in a structural context, which should enhance the data readability and provide additional clues for its interpretation.

In order to visualize the EEG findings in an anatomical context, we need also the spatial data of the cortex. MRI provides us with a possibility for imaging brain and other anatomical structures in great detail in a noninvasive way. Ideally, it should be possible to supplement the formidable spatial resolution of MRI with the unmatched temporal recording ability of EEG, to achieve vivid, expressive, comprehensive representations of brain activity, where both dimensions could be caught at the same time, at one glance, thus providing a possibil- ity for grasping and studying phenomena, which would otherwise escape our attention.

The difficulty of registering the recorded EEG with the subject's brain topography still remains though. One way of dealing with this problem is through the employment of artificial markers on the proband's head and the use of their recovered positions later for mapping of EEG findings on the proband's individual and highly specific cortical surface.

One point should be made exceedingly clear here. What we achieve by visualizing EEG findings on 3D-reconstructed cortex surfaces, is only one way of dealing with the data, it is by no way a reconstruction of the true relations or their only possible interpretation. In particular, it is decidedly misleading to attribute "raw" EEG measurements to the tissues lying directly underneath the respective electrode, but by visualizing higher-order, from many measurements e.g., statistically derived data we create a unified basis for comparison and interpretation.

We hope by presenting the EEG data as close as possible to its place of generation, to be able to uncover other important dependencies, e.g. mutual influences, or local correlations, which would be otherwise difficult if not impossible to notice.

\section{Problem}

The problem we tackled in this work was brought to us by a group of neurophysiologists [Petsche et al., Forschungsstelle Neurophysiologie und Neuroanatomie der Hirnrinde, ÖAW] striving to unambiguously assign observed and statistically evaluated EEGactivities to underlying cortical areas of the proband's cortex. The procedure employed previously by them was to represent these activities on schematic brain maps which was highly unsatisfying since such an important factor as the individual brain morphology had to be left out, and the identification of the participating cortical structures on a finer rather than a very coarse scheme showing only the brain lobes, had been out of the question. The obvious noninvasive way for obtaining the cortex morphology of healthy probands is through MRI and hence the task with which we were confronted was informally stated as follows:

"Find a method for combining the results of EEG and MRI studies, performed consecutively on the same subject in such a way that the EEG data be presented (visualized) directly on the proband's $3 D$-reconstructed cortex, thus providing a possibility for direct visual inspection and comparison of the evaluated EEG data in the context of their emergence."

After closer consideration, the task presented itself as consisting of three separate subproblems: 
1. Reconstruct the human brain surface.

2. Register the evaluated EEG data with 1.

3. Represent the EEG data from 2 on 1.

The rest of this work describes the concepts and solutions we developed for solving the above three subproblems.

\section{Concepts}

Our concept is based on the use of volume visualization techniques for reconstructing the brain surface (Subproblem 1), scattered data interpolation for calculating the functional maps of the EEG activities (Subproblem 2), and pseudo-coloring of the reconstructed cortex in accordance with a predefined color scale for visually representing the evaluated EEG-activities (Subproblem 3).

This approach has the advantage of preserving the 3D-effect of the extracted anatomical surfaces achieved through changes in the intensity of the surface voxels in the shading phase of the reconstruction process (1) and offering the possibility to experiment with different interpolation techniques in the registration phase (2). The representation phase (3) follows cleanly separated and allows to either calculate the desired color on-the-fly or employ a table-lookup (texture mapping) for speed and flexibility.

In the earlier stages of the project we favored an integrated solution [Thaller et al., 1991; Dimitrov, 1995], but soon had to realize that precalculating the texture maps is well worth the effort and offers additional possibilities not readily available otherwise, e.g., to precalculate the maps at a coarser resolution and then compare them in order to choose more promising ones for the lengthy process of the final interpolation and 3D-visualization.

Volume visualization is meanwhile a well-established tool in the scientific visualization community, but at the time our project started off it was relatively new and its use far from obvious. We settled for volume visualization by ray casting and grey level gradient shading, and had to solve numerous problems of conceptual and program-technical nature. The idea to use texture-mapping techniques for associating interpolated EEG-values with locations on the brain came up later in the project and proved the correctness of the choice of volume visualization in the first place.

The problems associated with the calculation of the functional maps are essentially those solved in the area of scattered data interpolation [Nielson, 1993]. Given that we start with a list of arbitrary locations (electrode positions) with assigned values (the results of the EEG analysis), it is clear that what we are looking for is a function (prescription, algorithm) assigning a unique value to every other place in its domain in such a way that certain restrictions hold true. We need a function which is at least $C^{0}$-continuous and localized in a sense that only the immediate electrode neighbors may contribute to the value in a chosen location. The first condition is immediately understandable, given that we try to interpolate a natural phenomenon, while the second becomes clear, when we consider the fact that we are going to represent/visualize the results from previous processing steps with as little distortion as possible.

These constraints led us directly to the concept of natural neighborhood and Delaunay triangulations which we exploited for developing a suitable scattered data interpolation method.

The pseudo-color representation of the pre- or onthe-fly calculated EEG values involves choosing a suitable mapping from the restricted real-valued functional EEG domain into a color-(sub)space. The choice of this mapping defines the expressive power and usefulness of the produced images for the purposes of comparative neurophysiological studies. We decided to map the interpolated EEG-values into the HSV color-space which accounts for ease of control and graphic quality.

\section{MATERIALS AND METHODS}

\section{Data acquisition}

The raw data gathered and used in the studies consist of EEG recordings and MRI scans performed on healthy individuals. The two data acquisition steps had to be performed consecutively, which posed the problem of marking and finding the positions of the EEG electrodes in the MRI scans. We solved this problem by use of artificial markers.

First, the EEG recording took place followed by an 3D-FLASH scan of the proband's head, on which the EEG electrodes were replaced by small Plexiglas capsules filled with the MRI contrast medium Gd-DTPAsolution (Magnevist/Schering) in a dilution of 1 : $1,000,000$. These dummy electrodes have the advantage of not producing large artifacts and visible distortions in the acquired MRI data set, but of still being clearly visible as bright spots in about five consecutive slices, so that their extraction and thus the approximate determination of the position of the actual EEG electrodes was made possible.

\section{EEG recordings}

The EEGs were recorded according to the international 10/20-system with respect to the averaged 
signals from both ear lobes. The equipment used was a 21-channel NIHON KOHDEN EEG-device. The probands had to perform mental tasks from a standardized test series involving mental arithmetic, memorizing pictures, listening to music, or simultaneous translations. Periods of activity alternated with such of rest. The data gathered covered the frequency range $(4-32 \mathrm{~Hz})$ of the usual frequency bands $-\alpha, \beta_{1}, \beta_{2}, \beta_{3}$, $\vartheta$. The results of this data acquisition step were time series of the signals sampled at a rate of 128 samples/ sec with a quantization of 12 bits/sample and a resolution of $240 \mathrm{nV} / \mathrm{bit}$.

\section{RI scans}

The raw volumetric data (slice stacks) for the experiments were obtained from a 1.5 Tesla MRI scanner (SIEMENS Magnetom 63). T1-weighted 3D-FLASH sequences were generated, delivering data sets of 128 horizontal slices each. Each slice had a resolution of 256 by 256 pixels (spatial resolution 0.88 by 0.88 $\mathrm{mm} / \mathrm{pixel}$ ), and a grey level resolution of $12 \mathrm{bits} /$ pixel. The scanning area was $157.6 \mathrm{~mm}$ in height, starting about $1 \mathrm{~cm}$ above the crown of the head (skin), and included the whole brain. This rendered the slice thickness approx. $1.24 \mathrm{~mm}$, with no gaps between successive slices. The net acquisition time was approximately $20 \mathrm{~min}$. The raw data per proband amounted to $128 * 256 * 256 * 2=16 \mathrm{MB}$.

\section{Data preparation}

\section{Spectral and statistical analysis}

The method applied for analyzing the ongoing EEG was based on signal processing by FFT and subsequent statistical analysis for uncovering significant differences between periods of mental activity and rest (1 min average duration) [Rappelsberger and Petsche, 1989]. The parameters frequency, location, power, and coherence were investigated, the latter with regard to neighboring or corresponding locations on both cerebral hemispheres.

In the past years, the method has been used to investigate a variety of cognitive tasks, such as listening to music, mental arithmetic, silent reading, visuospatial tasks, creative thinking, simultaneous interpretation.

The parameter coherence causes some problems at first. Coherence is a measure for the frequency correlation/phase consistency of two signals and gives hints at the functional coupling of the cortical regions where the signals originated. Basically, it is a property of two electrodes, so it can't be assigned to any of them without further considerations. The question arises how it could be incorporated in our representation scheme. One way of dealing with the problem would be by employing new, artificial locations, somewhere between the participating electrodes (what we did in [Thaller et al., 1991]), but this gives rise to somewhat cluttered, unnatural, difficult to interpret images.

Another approach would be to keep in each representation one electrode, the reference electrode for the representation, fixed and consider all the coherence values between that one and all the remaining electrodes. In that way, we would have a representation, and later a visualization, where the highest coherence values are observed at the reference electrode itself, which makes a sense, and the value at every other electrode could be seen in relation to this one.

Considering the fact that only the number of significant coherence changes (and not their absolute values) for each electrode between the period of activity and period of rest carries some information about the functional reaction of the underlying cortical regions, we decided to try and visualize only these changes in this work. The situation is comparable to that where a photographic picture is being taken with a fairly long exposure time from the top of a tower in a big city at night. On such a photo the search lights of the cars leave traces which may help identify the streets with the heaviest traffic and the most frequently used crossroads although the individual contributions of the cars are not visible any more. In a similar way, the purpose of the visualization discussed here was not to explore brief, repeatable, cognitive events but rather extended, singular mental acts with respect to the underlying cortical topography.

As output from this preparation step, we obtained a list of electrodes with corresponding values depicting in a concentrated form an interesting property (power or coherence) of the original raw EEG data, but only in a few arbitrarily scattered and otherwise unconnected locations around the proband's head, whose exact coordinates inside the MRI data set had yet to be determined.

\section{RI image pre-processing}

The raw data obtained in the MRI data acquisition step proved suitable for visualization by ray casting only after the application of several image processing operations. First, the 12 bits / pixel data was reduced to 8 bits/pixel by calculating a histogram of the grey values of the whole data set and using it for automati- 
cally choosing the appropriate center/width parameters for the re-quantization calculations. It was also necessary to re-sample the whole data sets in order to produce isotropic volumes for facilitating the subsequent voxel traversal. We experimented with different interpolation schemes and found that simple tri-linear interpolation suffices in most cases (since the inter-slice distance was quite small), and pre-processed our data accordingly. Only very basic filtering operations (arithmetic mean in a $3 \times 3$-neighborhood, median filter) were applied in some cases.

The most time-consuming and important preprocessing step proved to be the identification and segmentation of the anatomical structures to be visualized (cortex). Our experience showed that in most cases an automatic procedure is inappropriate or imprecise. We decided on a semi-automatic approach with control and guidance exercised by the human operator. The interactive program ISEG (developed by Dr. Miloš Šrámek) achieves this goal by providing the operator with a suite of logical and morphological operations in 3D and the means to apply them in an arbitrary order on the data set in question as well as to visualize and store the intermediate results (binary volume sets). In general, thresholding to highlight the brain tissue, erosion to disconnect it from surrounding structures, region filling to grow together the isolated parts and dilation to regain the tissue lost to the erosion are the processing steps required to segment the brain.

The result of this second preparation step is a corrected and enriched data volume, each voxel carrying additional information about its belonging to a structure, well-suited for volume visualization by means of ray casting and shading by grey level gradient shading.

\section{EEG and MRI data combination}

At this stage we had to combine the two previous data streams (EEG and MRI) into one, employing the localization information inherently present in the MRI data sets through the distortions caused by the artificial markers planted on the probands' heads pursuant to the procedure described in Data acquisition. For this purpose we used the program CUBE (developed by Dr. Igor Holländer) [Holländer and Šrámek, 1993; Dimitrov et al., 1994] allowing the interactive perfunctory visualization and examination of arbitrary volumetric data sets as well as the extraction of coordinates of any desired points inside them.
As a result, the list of EEG electrodes from Spectral and statistical analysis was augmented at this step with their respective positions in the MRI data set.

\section{Texture generation}

The EEG data as gathered and evaluated in the steps EEG recordings and Spectral and statistical analysis is not suited for direct representation on the 3Dreconstructed cortex. Up till now, it consists of just a few values assigned to discrete locations in the MRI data set. What we need is a function (prescription, algorithm), which would enable us to uniquely assign a meaningful value to each location on the reconstructed cortex. We could then use this function to calculate the required value whenever the rendering algorithm reaches a surface voxel in its shading phase and employ this value to obtain a representative color for the respective pixel, or we could precalculate and store a regular i.e., rectangular grid of values at a certain resolution, which could be easily looked up in the rendering phase. Both approaches rest on the possibility of finding a suitable interpolation function $F$, which assigns a "meaningful value" $F(V)$ to each $V$ from a predefined domain $D$. For the purpose of our presentation, we assume that the function $F$ exists and we know $n$ function values $F_{\mathrm{i}}$ in a set $V$ of discrete and unordered locations $V_{i} \in \mathrm{D}$, i.e., that we have at our disposal the set

$$
\mathrm{F}=\left\{F_{i}: F_{i}=F\left(V_{i}\right), V_{i} \in \mathrm{D}, i=1, \ldots, n\right\},
$$

where $D$ denotes the yet to be explained domain of definition of $F$. Our goal is to find a representation of $F$ which would enable its calculation in all locations $V \in$ D. Problems of this kind have come to be called scattered data interpolation problems, since the known data consists of an unordered (scattered) set of data value pairs $\left(V_{i}, F_{i}\right)$, and the task at hand is to find a function passing through (interpolating) them. The choice of the domain $D$ is given for one through the set of locations $V_{i}$, and through some implicit knowledge about the emergence of the values $F_{i}$ for another. In our case, we could assume that the function $F$ is defined in the whole 3D MRI data set, but this assumption would contradict our perception of the EEG signals as generated on the cortex, i.e., on a 2D surface. Empirical data of this kind are known as surface-on-surface data, and the associated interpolation problems as surface-onsurface interpolation problems, since the reconstructed function $F$ itself forms a surface over its domain $D$ (surface function). In our case, the cortical surface can be regarded as topologically equivalent to the unit 
sphere $U$, centered around some origin $\mathrm{O}$, which means that we can represent each location $V \in \mathrm{D}$ as $V=V(\varphi, \vartheta)$, where $\varphi$ and $\vartheta$ denote the spherical coordinates or direction of the location $V$ from the presumed origin $\mathrm{O}$. Thus, the scattered surface-onsurface data interpolation problem we face can be transformed to the search for a new function:

$$
G(\varphi, \vartheta)=F(V(\varphi, \vartheta)), 0 \leq \varphi \leq 2 \pi,-\frac{\pi}{2} \leq \vartheta \leq \frac{\pi}{2}
$$

where

$$
F\left(V_{i}\right)=F\left(V_{i}\left(\varphi_{i}, \vartheta_{i}\right)\right)=F_{i}=G\left(\varphi_{i}, \vartheta_{i}\right)
$$

holds true for all $V_{\mathrm{i}}$. The domain of definition of $G$ can be conveniently represented as

$$
U=\left\{(\varphi, \vartheta) \in[0 \ldots .2 \pi] \times\left[-\frac{\pi}{2} \ldots \frac{\pi}{2}\right]\right\} .
$$

\section{Restrictions and clarifications}

In order to specify further restrictions for the interpolation function $G=G(\varphi, \vartheta)$ aimed for, we have to consider its exact nature once more. It should approximate a natural phenomenon, i.e. the distribution of (evaluated) EEG signals on the human cortex, caused through some neurophysiological processes there. Natural processes tend to change continuously ("Natura non facit saltus," Linné, Philosophia botanica, 1751), so a natural restriction for the interpolating function $G$ is:

Restriction 1: $G=G(\varphi, \vartheta)$ should be at least $C^{0}$ continuous.

For the formulation of the next restriction, we considered the fact that $G$ had to be constructed in such a way as to assign all locations $V$ between the known locations $V_{i}$ meaningful values. If we turn the situation around, and assume the existence of $G$, the values $F_{i}$ become just samples (at a certain spatial frequency $f$ ) of a known continuous distribution $F$. Even if there were peaks of $F$ between the locations $V_{i}$ we could not hope to detect them at the given sampling frequency $f$. This leads us to:

Restriction 2: $G=G(\varphi, \vartheta)$ should be monotone between neighboring locations.

The last restriction for the interpolating function $G$ was dictated by the following considerations. First, we want to visualize the numerical results from the previous processing steps. So they should be changed/ distorted as little as possible in order to preserve the integrity of the data. Considering this, the most direct and non-falsifying way, would be to present only the data we have at our disposal, namely the values assigned to the electrodes themselves (by the way, we did exactly that in a previous work, see [Thaller et al., 1991], but this is not much worth for discovering global trends and dependencies. For such to become apparent, it is necessary to have gradual local changes which wouldn't destruct from the overall picture. The smoothest representations are achieved by global approximation and interpolation methods. But the use of such a method would be counterproductive to the wish not to distort the input data unnecessarily. So we decided to go the middle way and perform interpolation, but only locally, which we formalize in the last restriction:

Restriction 3: $G=G(\varphi, \vartheta)$ should be localized in the sense that each value $F(V)$ depends only on neighboring locations.

The notion of neighborhood, informally used in the formulation of restrictions 2 and 3 above, needs to be closer specified. The study of the ideas and problems involved leads to the concepts of Delaunay triangulations and Voronoi diagrams. A nice overview is presented by Aurenhammer [1991].

\section{Scattered data interpolation}

We are finally in a position to develop an algorithm for the interpolation of the surface function $G=$ $G(\varphi, \vartheta)$.

The question here is: Why do we need yet another interpolation method? Interpolation has been a major concern in the literature about EEG evaluation and mapping for at least 20 years. Some of the methods developed restrict the sites of the EEG electrodes to be located on a rectangular mesh [Darcey et al., 1980; Denoth et al., 1983], while others can handle arbitrary data locations on the scalp, but perform a global interpolation, e.g. they involve all of the given electrode locations in the calculations of a single interpolated value anywhere between the given electrodes [Thickbroom et al., 1984; Perrin et al., 1987]. The methods most commonly used are either four-nearest neighbors interpolation, or surface spline interpolation. The first one substitutes a fixed electrodes configuration and interpolates (bi-)linearly between the four nearest neighbors, which can result in map discontinuities, consequently a violation of our Restriction 1, while the second family tries to achieve a smooth value distribution surface by minimizing the bending energy of an infinite plate constrained to pass through the known points, which admittedly produces smooth surfaces, but also "false" extrema, namely such 
between the known locations which gravely violates our Restriction 2. That's why we decided to develop an own interpolation technique which could perhaps alleviate the shortcomings of the known and used ones.

Our primary idea was to precalculate the spherical Delaunay triangulation between the mapped electrode positions on the unit sphere and to use the so-gained neighbors' relation at the later volume rendering stage for interpolating a value for each brain surface voxel from the known values at its' natural neighbors' positions.

As it turned out later, interpolation and visualization could be easily and advantageously separated so that the interpolation function/algorithm would be used only for precalculating a regularly sampled map which could be later texture-mapped on the brain.

The interpolating algorithm we developed for the reconstruction of the surface function $G$ involves the following steps:

1. Map the set of 3D-sites (electrode locations) $V=$ $\left\{V_{\mathrm{i}}\right\}$ on the unit sphere $\mathrm{U}$ to obtain $\mathrm{V}^{\prime}=\left\{V_{\mathrm{i}}^{\prime} \in \mathrm{U}\right\}$.

2. Find the spherical Delaunay triangulation $D\left(V^{\prime}\right)$ for the set of spherical locations $V$ '.

3. For a spherical location $V^{\prime}=V^{\prime}(\varphi, \vartheta) \in U$ :

(a) Find the enclosing Delaunay triangle $\Delta A^{\prime} B^{\prime} C^{\prime}$ $\in D\left(\mathrm{~V}^{\prime}\right)$.

(b) Evaluate the interpolant $G_{\triangle \mathrm{A}^{\prime} B^{\prime} C^{\prime}}\left(V^{\prime}\right)=$ $\mathrm{w}_{\mathrm{A}}\left(V^{\prime}\right) G\left(A^{\prime}\right)+w_{\mathrm{A}}\left(V^{\prime}\right) G\left(B^{\prime}\right)+w_{\mathrm{A}}\left(V^{\prime}\right) G\left(C^{\prime}\right)$.

(c) Store the value $G_{\triangle A^{\prime} B^{\prime} C^{\prime}}\left(V^{\prime}\right)$ for later use.

Step 1 is easily achieved through the well-known transformation from Cartesian $(x, y, z)$ to spherical $(\varphi, \vartheta, r)$ coordinates:

$$
\begin{aligned}
& \varphi=\left\{\begin{array}{rr}
\arccos \frac{x}{\sqrt{x^{2}+y^{2}}}+2 k \pi & y \geq 0 ; x^{2}+y^{2} \neq 0 \\
-\arccos \frac{x}{\sqrt{x^{2}+y^{2}}}+2 k \pi & y<0 ; x^{2}+y^{2} \neq 0 \\
\text { undefined } & x^{2}+y^{2}=0
\end{array}\right. \\
& \vartheta=\left\{\begin{array}{cr}
\arcsin \frac{z}{\sqrt{x^{2}+y^{2}+z^{2}}} & x^{2}+y^{2}+z^{2} \neq 0 \\
\text { undefined } & x^{2}+y^{2}+z^{2}=0
\end{array}\right. \\
& \mathrm{r}=\sqrt{x^{2}+y^{2}+z^{2} .}
\end{aligned}
$$

Dropping the radius $r$ (and implicitly substituting 1 for

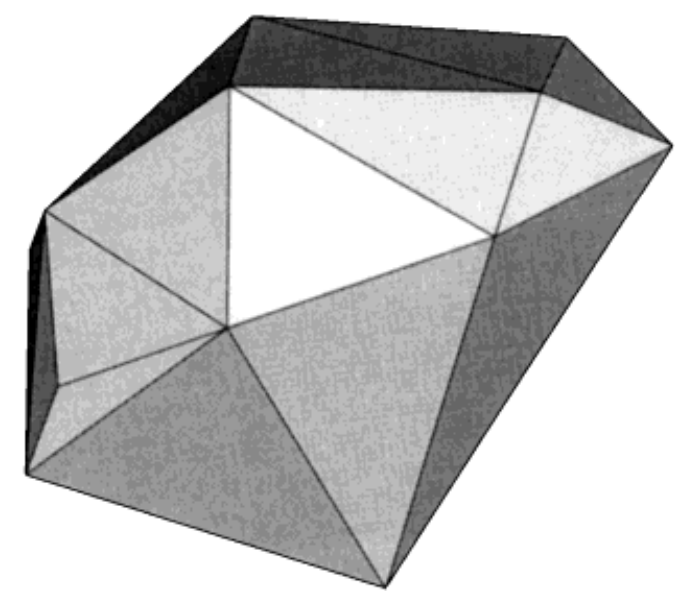

Figure 1.

Convex hull of the EEG-electrodes' positions.

it), we reach our goal of mapping $V$ to a set $V^{\prime}$ on the unit sphere $U$.

Step 2 of our interpolation algorithm calls for the construction of the spherical Delaunay triangulation $D\left(V^{\prime}\right)$ of the site set $V^{\prime}$ (actually, the site set $V^{\prime} \cup\{W\}$, where $W$ is an auxiliary site positioned in such a way as to allow the convenient closure of $V^{\prime}$ ). Figure 1 depicts a set of EEG-electrodes rendered as a polygonally bounded object defined by means of its convex hull.

Basically, two ways for achieving the triangulation exist: either directly triangulating the point set $V^{\prime}$ in spherical space by substituting the geodesic distance $d(A, B)=\arccos (A, B), \forall A, B \in \mathrm{U}$, or triangulating the convex hull of $\mathrm{V}^{\prime}$ in three-dimensional Euclidean space. Both methods are equivalent in terms of output, but differ in running time requirements. Considering the cardinality of the data sets we are dealing with $(\approx 20)$, this can be neglected since the two methods showed no measurable differences. An example of a spherical Delaunay triangulation (together with the underlying convex hull) is shown in Figure 2.

In Step 3 we utilize the parameter representation of the domain of definition of $G$ (Eq. 4) and discretize it at a given resolution $\mathrm{M} \times \mathrm{N}$ by setting

$$
\begin{aligned}
V^{\prime}\left(\varphi_{i}, \vartheta_{i}\right)=V^{\prime}\left(\frac{i}{N} 2 \pi,-\frac{\pi}{2}+\frac{j}{N} \pi\right), \\
\quad i=1, \ldots, M, j=1, \ldots, N
\end{aligned}
$$

For explaining Step 3a, we consider the situation as illustrated in Figure 3. 


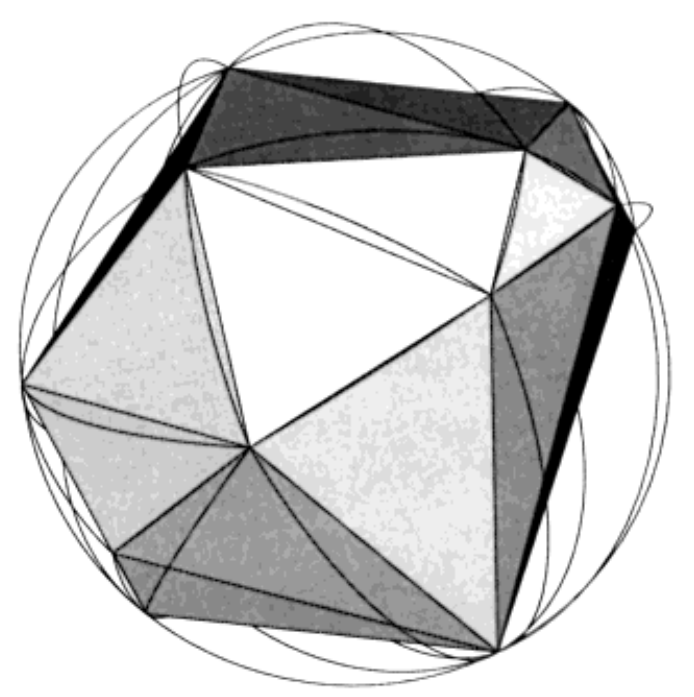

Figure 2.

Spherical D elaunay triangulation.

Here $A, B$, and $C$ denote three vertices in general position being projected onto the unit sphere $U$ which is centered at the centroid (center of gravity) $O$ of all vertices. Their projection sites shall be $A^{\prime}, B^{\prime}$, and $C^{\prime}$, respectively. The spherical triangle $\Delta A^{\prime} B^{\prime} C^{\prime}$ forms a part of the spherical Delaunay triangulation $D\left(\mathrm{~V}^{\prime}\right)$ of the set of mapped sites $V^{\prime}$ as determined in Step 2. The point $V$ represents a voxel on the reconstructed brain surface. After mapping $V$ to the unit sphere, it takes position $V^{\prime}$ inside the Delaunay triangle $\Delta A^{\prime} B^{\prime} C^{\prime}$ (Step 3a). Henceforth the sites $A^{\prime}, B^{\prime}, C^{\prime} \in V^{\prime}$ are considered the natural neighbors of $V^{\prime}$.

Once the neighbors of a site are known, an interpolated surface function value for that site can be calculated from the values for its neighbors by evaluating the following simple formula (Step 3b):

$$
\begin{aligned}
G_{\Delta A^{\prime} B^{\prime} C^{\prime}}\left(V^{\prime}\right)= & w_{A}\left(V^{\prime}\right) * G\left(A^{\prime}\right)+ \\
& w_{B}\left(V^{\prime}\right) * G\left(B^{\prime}\right)+w_{C}\left(V^{\prime}\right) * G\left(C^{\prime}\right) .
\end{aligned}
$$

Here, $\mathrm{w}_{\mathrm{A}}, \mathrm{w}_{\mathrm{B}}$, and $\mathrm{w}_{\mathrm{C}}$ are yet-to-be-found real-valued weight functions, and $G\left(A^{\prime}\right), G\left(B^{\prime}\right), G\left(C^{\prime}\right)$ denote (through Eq. 3) the observed function values (obtained EEG values at the positions of vertices $A, B$, and $C$, respectively).

Initially, the interpolant $G_{\triangle A^{\prime} B^{\prime} C^{\prime}}$ calculates the surface function $G$ only for sites projected inside the triangle $\Delta A^{\prime} B^{\prime} C^{\prime}$. Through the generalization

$$
G\left(V^{\prime}\right)=\underset{\Delta A^{\prime} B^{\prime} C^{\prime} \in D\left(V^{\prime}\right)}{\cup} G_{\Delta A^{\prime} B^{\prime} C^{\prime}}\left(V^{\prime}\right),
$$

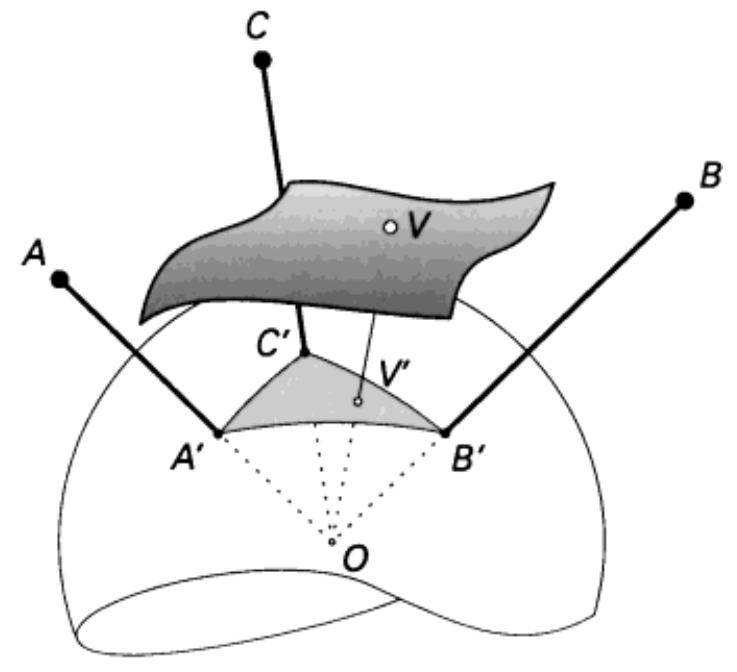

Figure 3.

Global interpolation situation.

we get a representation for the surface function $G$, defining it in the whole domain $D$.

The weight functions $w_{\mathrm{A}}, w_{\mathrm{B}}, w_{\mathrm{C}}$ in Eq. 6 are real-valued weights depending on the position of $V^{\prime}$ inside the Delaunay triangle $\Delta A^{\prime} B^{\prime} C^{\prime}$. They should be chosen in such a way as to satisfy the continuity (1) and monotony (2) restrictions for the surface function $G$ inside the regarded triangle $\Delta A^{\prime} B^{\prime} C^{\prime}$ and to guarantee its continuity by the transition from one Delaunay triangle to the other. The relations in the Delaunay triangle $\Delta A^{\prime} B^{\prime} C^{\prime}$ are represented in Figure 4.

The points $A^{\prime \prime}, B^{\prime \prime}$, and $C^{\prime \prime}$ shall be the intersection points of the lines (great circles) passing through $V^{\prime}$ and connecting one triangle vertex with the opposite edge in the regarded Delaunay triangle. They are found as intersections of two great circles on the unit sphere.

It is intuitively clear that the weights $w_{\mathrm{A}}, w_{\mathrm{B}}$, and $w_{\mathrm{C}}$ should be at their highest in the vicinity of the vertices $A^{\prime}, B^{\prime}$, and $C^{\prime}$, since they determine the influence of the respective empirical value on the interpolated surface

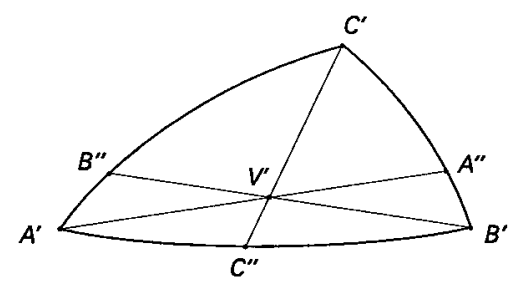

Figure 4.

The relations in a D elaunay triangle. 
function value. If we could choose the weights in such a way as to satisfy the conditions

$$
\begin{aligned}
& w_{A}\left(A^{\prime}\right)=1, \quad w_{A}\left(A^{\prime \prime}\right)=0, \quad w_{A} \text { monotone on } A^{\prime} A^{\prime \prime}, \\
& w_{B}\left(B^{\prime}\right)=1, \quad w_{B}\left(B^{\prime \prime}\right)=0, \quad w_{B} \text { monotone on } B^{\prime} B^{\prime \prime}, \\
& w_{C}\left(C^{\prime}\right)=1, \quad w_{C}\left(C^{\prime \prime}\right)=0, \quad w_{C} \text { monotone on } C^{\prime} C^{\prime \prime},
\end{aligned}
$$

then we could guarantee also that the reconstructed surface function $G$ passes through its points of support and is continuous and monotone between them as previously required. The continuity of the interpolating function on the edges of the Delaunay triangles becomes obvious if we consider the fact that the influence of the non-incident vertex, which we can assume to be $A$ without loss of generality, is effectively eliminated through the condition $w_{\mathrm{A}}\left(A^{\prime \prime}\right)=0$.

A set of weight functions fulfilling the above conditions is given by the equations:

$$
\begin{aligned}
& w_{A}\left(V^{\prime}\right)=1-\frac{\mathrm{d}\left(\mathrm{A}^{\prime}, \mathrm{V}^{\prime}\right)}{d\left(A^{\prime}, A^{\prime \prime}\right)}, \\
& w_{B}\left(V^{\prime}\right)=1-\frac{\mathrm{d}\left(\mathrm{B}^{\prime}, \mathrm{V}^{\prime}\right)}{d\left(B^{\prime}, B^{\prime \prime}\right)}, \\
& w_{C}\left(V^{\prime}\right)=1-\frac{\mathrm{d}\left(\mathrm{C}^{\prime}, \mathrm{V}^{\prime}\right)}{d\left(C^{\prime}, C^{\prime \prime}\right)} .
\end{aligned}
$$

Here, $d(A, B)$ denotes again the geodesic distance between two points $A, B \in \mathrm{U}$.

\section{Value-to-color mapping}

The interpolation surface function $G\left(V^{\prime}\right)$ as defined in Eq. 7 enables the evaluation of representative and meaningful real values $G \in F$ for all locations in its domain $U$. The discretization of $U$, employed in Step 3 , allows also for their efficient storage and retrieval. Their visual representation, however, calls for an additional mapping $m: F \rightarrow C$ from the functional domain $F$ of $G$ into a color-space $C$. We achieve $m$ in a two-step procedure:

First, we normalize $G$ through the transformation:

$$
n: F \rightarrow[0 . .1], n\left(G\left(V^{\prime}\right)\right)=\frac{G\left(V^{\prime}\right)-\min (G)}{\max (G)-\min (G)}
$$

Considering Restriction 2, we can substitute $\min (G)=$ $F \min$ and $\max (G)=F \max ($ see Eq. 1) which allows us to rewrite Eq. 8 in the form:

$$
n: G \rightarrow[0 . .1], n\left(G\left(V^{\prime}\right)\right)=\frac{G\left(V^{\prime}\right)-F_{\min }}{F_{\max }-F_{\min }}
$$

where $F_{\min }$ and $F_{\max }$ denote the smallest, resp. largest empirically observed values in $F$.

In the second step, we employ a transformation

$$
t:[0 . .1] \rightarrow \mathrm{HSV}
$$

into the HSV-color-space by setting

$$
\begin{gathered}
H\left(n\left(G\left(V^{\prime}\right)\right)\right)=n\left(G\left(V^{\prime}\right)\right) \\
S\left(n\left(G\left(V^{\prime}\right)\right)\right)=n\left(G\left(V^{\prime}\right)\right) \\
V\left(n\left(G\left(V^{\prime}\right)\right)\right)=1
\end{gathered}
$$

i.e., we map the interpolated value $G$ to a color by allowing for the hue $H$ and saturation $S$ to change simultaneously and rendering them at the highest available intensity $(V=1)$. Utilizing the discretization from Step 5, we store (Step 3c) the calculated $(H, S, V)$ triple in a rectangular map of dimension $M \times N$. In this EEG-activity map, we recognize areas of relatively high change in activity as colorful spots and such of relatively low or no activity as uniformly white spots, subject only to intensity changes by the shading procedure in the rendering/texture-mapping process.

\section{Volume rendering}

We decided to use volume rendering by ray casting and grey level gradient shading [Höhne et al., 1990] for visually representing the brain from the MRI data acquired for each proband. This choice enables the control over each voxel of the reconstructed surface (cortex) and allows us to experiment with different representations of the measured/evaluated EEG data. We developed our own rendering package VORTEX, since no commercially/publicly available option at that time could satisfy our requirements for control over the different passes of the rendering process, for robustness and quality of the results.

\section{General decisions}

Input to the program consists of eight-bit isotropic data stacks, enhanced with one-bit segmentation information denoting in each voxel its belonging to an 
object. User control is achieved through supplying the usual viewing, shading and color information. Rays are cast through each pixel of the output image and incrementally stepped through the scenery, stopping whenever a voxel belonging to an object is reached. At this stage, the grey level gradient in the voxel is substituted for the object's normal, the user supplied coloring information is fetched, and a shading procedure is evaluated rendering a color value for the pixel in question.

\section{Optimizations}

A number of problems with the volume rendering algorithm as sketched above exist. All of them are concerned either with its efficiency or with the quality of the produced results (images). The most severe problems and our solutions for them are:

1. Antialiasing is achieved through adaptive oversampling,

2. Localization of the intersected surfaces is refined by trilinear interpolation (selectively in the binary or grey level volumes),

3. Shading is improved by employing better gradient approximations then first-order finite differences,

4. Voxel traversal is accelerated by means of variable step distances.

\section{Texture mapping}

The presence of the clearly defined coloring step in the rendering algorithm enables the substitution of any desired coloring source. We employ a texture mapping procedure which calculates first a direction $(\varphi, \vartheta)$ from the given surface voxel coordinates $(x, y, z)$. By means of the parameters $\varphi$ and $\vartheta$ a color triple $(H, S, V)$ is fetched from a pre-calculated user-supplied texture map. In order to minimize aliasing effects, a bi-linear interpolation in the $(\varphi, \vartheta)$-parameter space is performed.

\section{RESULTS}

The result of our endeavors in the framework of the presented project consists of a suite of programs well-suited for solving:

1. Scattered data interpolation of surface-on-surface functions,

2. Pseudo-colored visualization of surface-on-surface functions,
3. Visualization of volumetric data containing welldefined surfaces (medical imaging data),

4. Brain visualization with EEG data mapping.

We demonstrated the usefulness of our visualization software by employing it to represent the mutual functional electrical relationships between different brain regions as detected by EEG coherence analysis.

Our programs are further employed in studies concerned with visualizing areas of high/low activation on the cortex during processes of solving standardized mental tasks.

\section{Program packages}

\section{APEX}

MAPEX is the program which achieves the scattered data interpolation between a set of locations (EEG electrodes) with assigned values (EEG evaluations). It is realized as a C-program with a command-line interface. Input to the program consists of a file containing the names, coordinates and assigned values of the vertices to be interpolated, and a second file defining the triangles of the required Delaunay triangulation. Numerous options controlling the program behavior (e.g., different interpolation methods for test purposes, choice of color subspace, output image size) can be supplied. The output from the program is an image representing the interpolated function in the $(\varphi, \vartheta)$-domain (latitude, longitude) by means of a color coding. This can be supplied to the volume-renderer VORTEX for texturing onto a 3D-reconstructed object (cortex).

\section{C ++ -class library}

VORTEX was implemented as the $\mathrm{C}++$-class library libvv.a. The objects implemented by the library libvv.a can be directly included and manipulated in a $\mathrm{C}++-$ program providing the necessary parameters. A simple $\mathrm{C}++$-program making use of the library is shown in Figure 5 in the Appendix.

\section{Tcl-interface}

The direct use of the $\mathrm{C}++$-class library is possible and pretty straightforward, but it still requires a few passes of the edit/compile/run cycle. In order to facilitate its use, especially for repetitive production tasks, we supplied it with a scriptable command interface built around the publicly available Tcl-library. Our extension is realized as the add-on package libv.a, 


\section{APPENDIX}

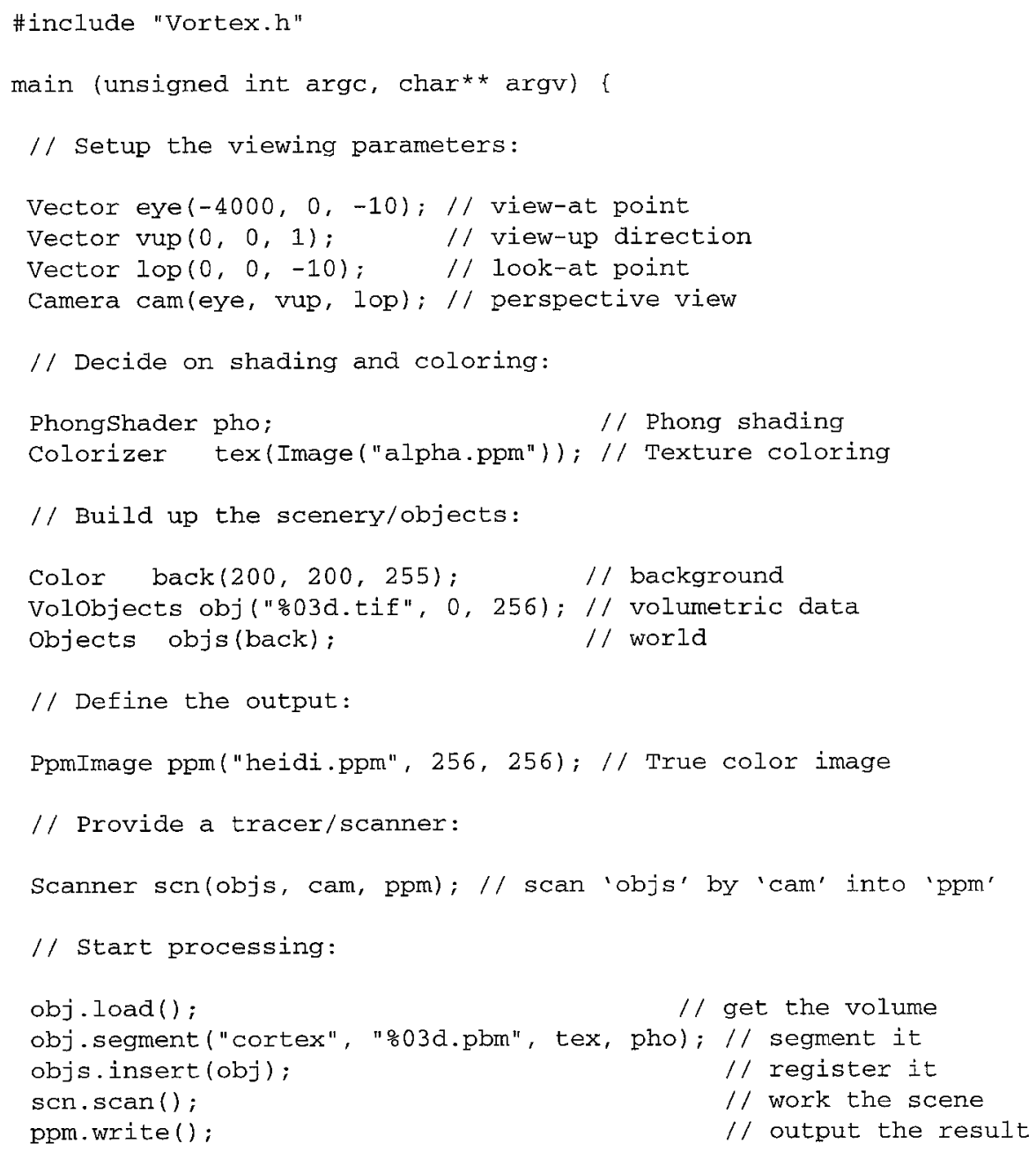

Figure 5.

A simple $\mathrm{C}++$-program demonstrating the use of the VO RTEX visualization library.

which when linked together with the libtcl.a extends the command set of Tcl by commands for creating and manipulating VORTEX-objects and yields the command-line interpreter vvsh. Since the original data definition and control structures of $\mathrm{Tcl}$ are retained, they can be employed advantageously for creating more complex scenarios in a visualization situation, e.g., fly-arounds, walk-throughs or other animation sequences. To illustrate the use of the interpreter vvsh, we show in Figure 6 the script which solves the same task as the sample $\mathrm{C}++$-program from Figure 5. Based on the interpretive nature of the scripting language and the ease of manipulation of the original (Tcl) and additional (VORTEX) objects and their seamless integration into one program (vvsh), the addition of loops and/or new objects for producing animation sequences of arbitrary length or complexity is made fast and easy. A few examples can be viewed on the World Wide Web at the URL: /http://www.iinform.oeaw. ac.at/ leon/videos). 


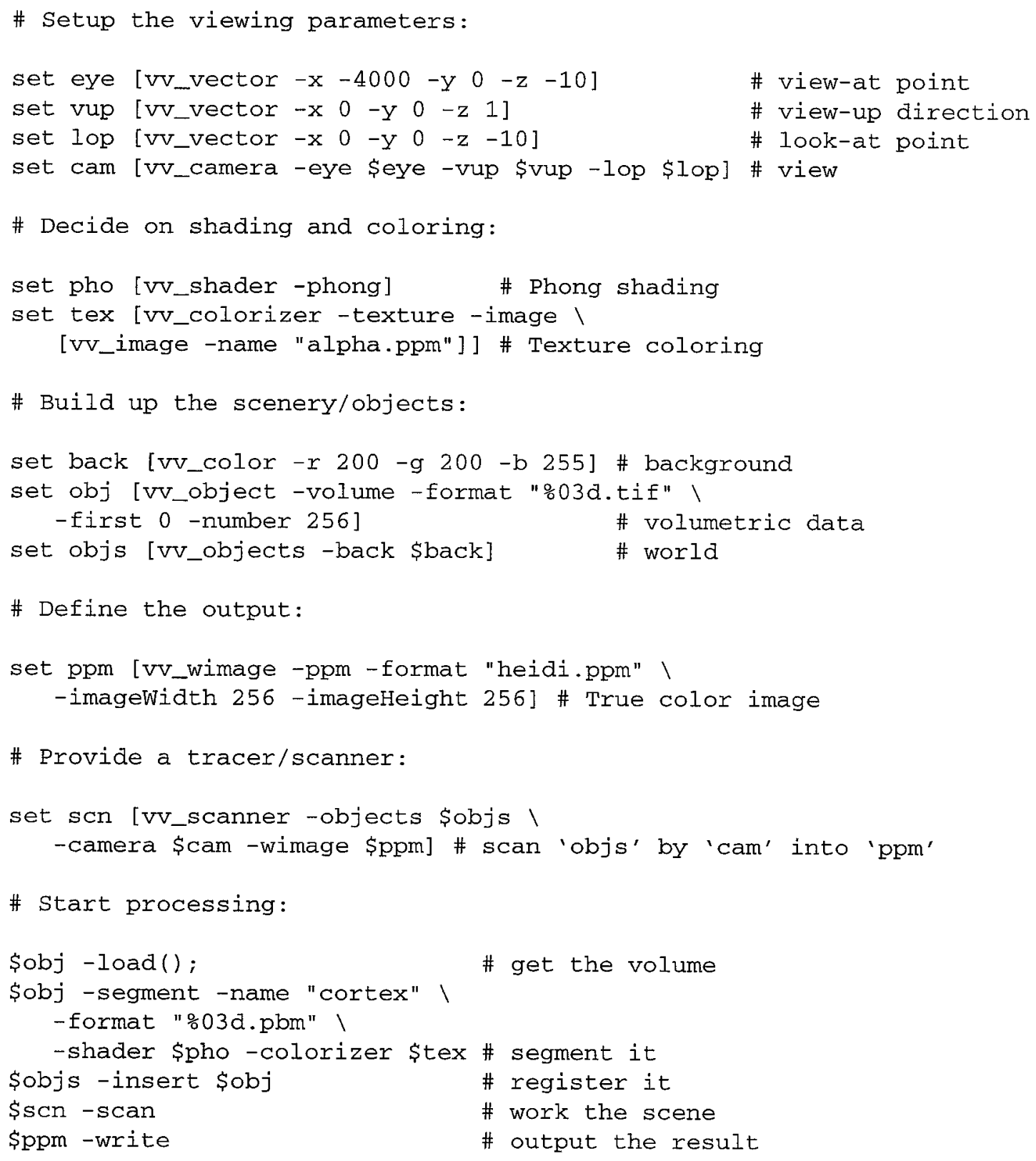

Figure 6.

A sample vvsh-script, demonstrating the interactive use of the library.

\section{Combined representations of EEG and MRI data}

Our method and its software realization VORTEX allow for better attribution of observed/evaluated EEG-activities to the supposedly involved cortical areas and their direct representation there. The visual inspection by experts and the improved spatial visualization facilitate the formation of more specific hypotheses about the distribution of participating cortical areas during cognitive tasks than was previously possible. The unsurpassed human pattern matching ability can be thus advantageously put to work, and we hope that new, previously unnoticed relations and dependencies will emerge. Our intention is to first visualize in this new way all the data we gathered in experiments over the last years and compare the results with the already obtained ones, hoping for new insights. [For an in-depth discussion of the coherence changes method and an interpretation of some data gained by it see e.g., Holländer et al., 1997.] 


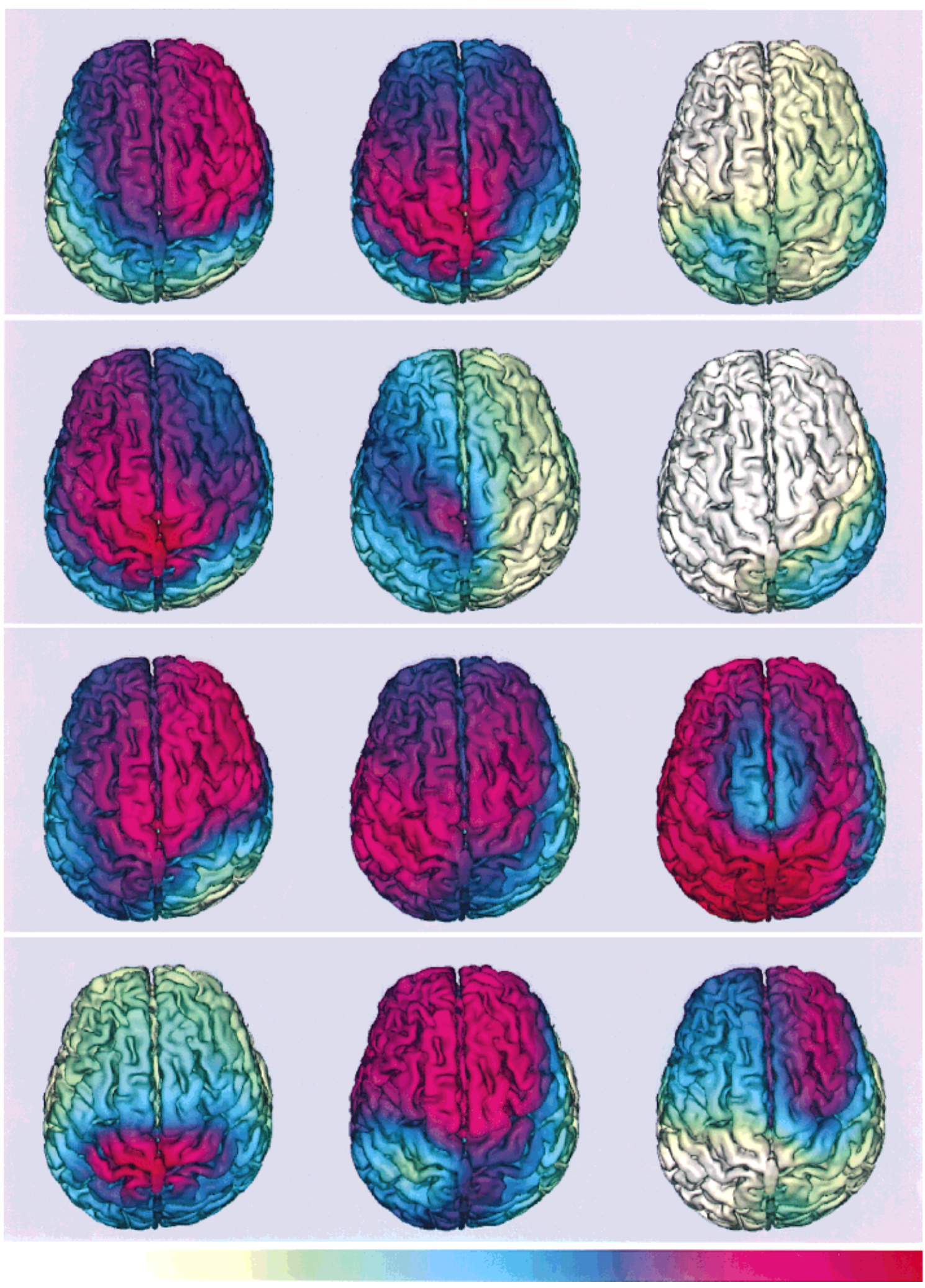

Figure 7.

EEG coherence distribution interpolated by MAPEX and mapped by VO RTEX. Tasks from top to botto m: Mental calculation, Listening to music, Memorizing music, Reading a score. Frequency bands from left to right: $\alpha: 7.0-12.5 \mathrm{~Hz}, \beta_{1}: 13.0-18.0 \mathrm{~Hz}, \beta_{2}: 18.5-31.5 \mathrm{~Hz}$. 
A few examples (images) generated by our package from data we acquired in our experiments over the past few years are shown in Figure 7 of the Appendix. The images present top views of the 3D-reconstructed brain of a right-handed 68-year-old male musician with superimposed interpolated, pseudo-colored coherence changes maps during the performance of four standardized mental tasks in three chosen frequency bands with respect to the averaged EEG at rest. We visualized the number of significant coherence changes (increases and decreases) at each electrode to all the remaining ones with respect to the corresponding coherences during a reference EEG period of rest. The color coding scale ranging from minimal to maximal coherence changes is shown at the bottom. Using such representations, an identification of the involved cortical structures during the mental act is feasible.

Combining many such representations taken at different stages along the temporal axis, an animated visualization sequence (video) of the mental activity can be achieved, which could be helpful for discovering trends and dependencies which would pass unnoticed otherwise. Examples of such videos can be seen on the World Wide Web at the URL: /http://www. iinform.oeaw.ac.at/ leon/videos.

At present, we are busy applying our visualization method to the data we gathered over the years and trying to view and systemize the results. We hope to be able to show in the next future, that this technique effectively allows neurophysiologists to determine whether the EEG differences between subjects are due in part to individual brain morphology.

\section{ACKNOWLEDGMENTS}

Our thanks belong by right to Hellmuth Petsche from the Research Department Physiology and Anatomy of the Cortex of the Austrian Academy of Sciences for initiating this research as well as for consolidating and evaluating the final results. Without him neither the motivation, nor the encouragement for further endeavors would have arisen. Mr. Reinhard Thaller, affiliated at that time with the Institute of Information Processing of the Austrian Academy of Sciences, conducted the initial studies and pointed out the way for further development. Mr. Miloš Šrámek from the Institute for Measurement of the Slovak Academy of Sciences provided valuable contributions especially to the area of volume rendering and its acceleration by means of digital distance transforms. Mr. Igor Holländer developed the program CUBE and contributed valuable suggestions in many fruitful discussions.
The MRI scans were performed at the General Hospital of Vienna (AKH), the EEG recordings and their subsequent analysis at the Institute of Neurophysiology of the University of Vienna.

\section{REFERENCES}

Aurenhammer F (1991): Voronoi diagramms - a survey of a fundamental geometric data structure. ACM Comput Surveys 23(3):345-405.

Darcey TM, Ary JP, Fender DH (1980): Spatiotemporal visually evoked scalp potentials in response to partial-field patterned simulation. Electroencephalogr Clin Neurophysiol 50:348-355.

Denoth F, Rappoli R, Bruni I, Navona C, Nencioni C (1983): Equipotential line map representation and temporal evolution of multichannel recorded cnv activity. In: Seventh Int. Conf. on Event Related Potentials of the Brain: Epic VII, Florence, Italy. Università degli Studi di Firenze and Consiglio Nazionale delle Recerche, pp. 4-10.

Dimitrov LI (1995): Pseudo-colored visualization of EEG-activities on the human cortex using MRI-based volume rendering and Delaunay interpolation. In: Kim Y (ed): Medical Imaging 1995: Image Display, Vol. Proc. SPIE 2431, San Diego, California. SPIE-The International Society for Optical Engineering, pp. 460-469.

Dimitrov LI, Holländer I, Šrámek, M (1994): Visualizing different kinds of 3D data sets in a uniform and comprehensive manner. In: Kuznetsov N, Soyfer V (eds): Image Processing and Computer Optics (DIP-94), Vol. Proc. SPIE 2363, Samara, Russia. SPIE-The International Society for Optical Engineering, pp 222-229.

Gevins AS, Le J, Brickett P, Reutter B, Desmond J (1991): Seeing through the skull: Advanced eegs use mris to accurately measure cortical activity from the scalp. Brain Topography 4:125-131.

Gevins AS, Le J, Martin N, Brickett P, Desmond J (1994): High resolution eeg: 124-channel recording, spatial enhancement, and mri integration methods. Electroencephalogr Clin Neurophysiol 90:337-358.

Höhne KH, Bomans M, Pommert A, Riemer M, Schiers C, Tiede U, Wiebecke G (1990): 3D Visualization of tomographic volume data using the generalized voxel model. Vis Computer 6(1):28-36.

Holländer I, Petsche H, Dimitrov LI, Filz O, Wenger E (1997): The reflection of cognitive tasks in eeg and mri and a method of its visualization. Brain Topography 9(3).

Holländer I, Šrámek M (1993): An interactive tool for manipulation and presentation of 3D tomographic data. In: Lemke HU, Inamura K, Jaffe CC, Felix R (eds): Computer Assisted Radiology '93, Berlin. Springer-Verlag, pp. 378-383.

Nielson GM (1993): Modeling and visualizing volumetric and surface-on-surface data. In Hagen $\mathrm{H}$, Müller H, Nielson GM (eds): Focus on Scientific Visualization, Springer, Berlin Heidelberg, pp. 191-242.

Nunez PL (1995): Neocortical Dynamics and Human EEG Rythms. Oxford University Press.

Perrin F, Pernier J, Bertrand O, Giard MH, Echallier JF (1987): Mapping of scalp potentials by surface spline interpolation. Electroencephalogr Clin Neurophysiol 66:75-81.

Rappelsberger P, Petsche H (1989): Probability mapping: power and coherence analysis of cognitive processes. Brain Mapp 1:46-54.

Thaller RA, Petsche H, Rappelsberger P, Pockberger H, Lindner K, Imhof H (1991): An approach to a synopsis of EEG parameters, morphology of brain convolutions and mental activities. Brain Topography 4(1).

Thickbroom GW, Mastaglia FL, Carroll WM (1984): Spatiotemporal mapping of evoked cerebral activity. Electroencephalogr Clin Neurophysiol 59:425-431. 